\title{
Impact of the Time to Chemoradiation Initiation in Patients with Glioblastoma
}

\author{
Abdullah Alhumiqani ${ }^{1 *}$, Bassam Basulaiman ${ }^{1}$, Ashraf Elyamany $^{3}$, Ali Balbaid² and Abdullah K Altwairgi ${ }^{1}$ \\ ${ }^{1}$ Department of Medical Oncology, Comprehensive Cancer Center, King Fahad Medical City, Riyadh, Saudi Arabia \\ ${ }^{2}$ Department of Radiation Oncology, Comprehensive Cancer Center, King Fahad Medical City, Riyadh, Saudi Arabia \\ ${ }^{3}$ Department of Medical Oncology, King Saud Medical City, Riyadh, Saudi Arabia/SECI, Assiut University, Egypt
}

\begin{abstract}
Background and purpose: Glioblastoma is the most common malignant primary brain tumor. The current standard therapy consists of surgical resection followed by concurrent chemoradiotherapy (CCRT) and adjuvant chemotherapy using temozolomide (TMZ). Treatment is typically initiated within 4 to 6 weeks. We aimed to investigate the effect of the time to CCRT initiation in a cohort of patients with glioblastoma.
\end{abstract}

Materials and methods: All adult patients with glioblastoma treated at the King Fahad Medical City (KFMC) between January 2008 and May 2016 were included. The time to CCRT initiation was defined as the time interval between surgery and the commencement of CCRT.

Results: A total of 115 patients were diagnosed with glioblastoma during the study period; of these, 99 patients were eligible for analysis. The median time to CCRT initiation was 5.1 weeks. Twenty-one percent of patients received CCRT within four weeks after surgery, 32\% between 4 and 6 weeks after surgery, and around $47 \%>6$ Weeks after surgery. The median progression- free survival (PFS) of these groups was 11.2 months, 7.6 months, and 9.2 months, respectively; their median overall survival (OS) was 25 months, 16.9 months, and 20.1 months, resp-ectively. However, in the multivariate analysis, the time to initiation of CCRT did not show a significant association with OS.

Conclusion: In our study, the time to the initiation of CCRT did not have an impact on the survival outcome. Prospective studies are needed to define the optimal timing of CCRT after surgical resection in patients with glioblastoma.

Keywords: Glioblastoma; Chemotherapy; Radiotherapy; Survival

\begin{abstract}
Abbreviations: CCRT: Concurrent Chemoradiotherapy; ECOG: Eastern Cooperative Oncology Group; KFMC: King Fahad Medical City; MGMT: O6-Methylguanine-DNA-Methyltransferase; OS: Overall Survival; PFS: Progression-Free Survival; PS: Performance Status; TMZ: Temozolomide; RT: Radiotherapy
\end{abstract}

\section{Introduction}

Glioblastoma is the most common and most malignant primary brain tumor in adults. Despite advances in modern surgical and adjuvant therapies, glioblastoma remains a challenging disease entity. The standard of care in patients with these tumors includes maximal surgical resection, followed by radiotherapy (RT) with concomitant and adjuvant temozolomide (TMZ). The addition of TMZ to RT has increased both median survival (from 12.1 months to 14.6 months) and the 2- year survival rate (from $10 \%$ to $26 \%$ ) in patients with glioblastoma [1]. Although different prognostic groups can be distinguished (e.g., by using the recursive partitioning analysis classification developed by the Radiation Therapy Oncology Group consortium), the overall prognosis of glioblastoma remains poor [2-4]. Studies have demonstrated a negative effect of delayed RT or prolonged treatment duration, mostly in head and neck squamous cell carcinoma and lung cancer, but also in breast and prostate cancer [5-7]. Thus, the presence of a treatment- related time factor in aggressively proliferating glioblastoma is likely; however, evidence on the effect of the overall duration of chemoradiotherapy on clinical outcomes has been limited and inconclusive [8-10]. In the context of glioblastoma, the association between delaying CCRT and the outcome is less clear, though some studies have demonstrated an association between a delay in CCRT and poor survival [11-13]. Therefore, this retrospective analysis aimed to investigate the effect of the time to therapy initiation in a contemporary cohort of patients with glioblastoma treated with RT and concomitant adjuvant TMZ in Saudi Arabia.

\section{Materials and Methods}

\section{Patients and data collection}

We conducted a single- center retrospective cohort study at the Comprehensive Cancer Centre of the King Fahad Medical City (KFMC) in Riyadh, Saudi Arabia. All adult patients ( $\geq 18$ years old) who were diagnosed with histologically confirmed glioblastoma between January 2008 and June 2016 (identified in the pathology database of the KFMC) and who received concurrent chemoradiotherapy (CCRT) were included. Data were obtained from patient charts and electronic medical records. A data collection form was developed to collect data on patient demographics, pathology, details of RT and chemotherapy, as well as progression and survival outcomes.

The study proposal was approved by the Institutional Review Board of the KFMC. The work described herein has been carried out in

${ }^{*}$ Corresponding author: Dr. Abdullah Alhumiqani, Department of Medical Oncology, Comprehensive Cancer Center, King Fahad Medical City, Riyadh, Saudi Arabia,Tel: +966112889999; E-mail: alhumiqani05@yahoo.comm

Received July 26, 2018; Accepted July 31, 2018; Published August 06, 2018

Citation: Alhumiqani A, Basulaiman B, Elyamany A, Balbaid A, Altwairgi AK (2018) Impact of the Time to Chemoradiation Initiation in Patients with Glioblastoma. J Integr Oncol 7: 209. doi: 10.4172/2329-6771.1000209

Copyright: ( $) 2018$ Alhumiqani A, et al. This is an open-access article distributed under the terms of the Creative Commons Attribution License, which permits unrestricted use, distribution, and reproduction in any medium, provided the original author and source are credited. 
accordance with The Code of Ethics of the World Medical Association (Declaration of Helsinki). Informed patient consent was not required because of the study's retrospective design.

\section{Measures and definitions}

The time for therapy initiation was defined as the time interval between definitive surgery and the commencement of RT and concurrent TMZ. Progression- free survival (PFS) was defined as the time from the histological diagnosis of glioblastoma until first progression or death, whichever occurred first. If neither event was observed, the patient was censored at the date of the last follow-up examination. Similarly, overall survival (OS) was defined as the time from the histological diagnosis of glioblastoma to the date of death of any cause. Patients who were not reported dead or lost to follow-up were censored at the date of the last follow-up examination.

\section{Statistical Analyses}

The patients were classified by time intervals, based on the time to CCRT initiation after surgery. Specifically, the intervals of $<4$ weeks, 4-6 weeks, and $>6$ weeks were selected, based on the distribution of the intervals in percentiles (25th, 50th, and 75th, respectively). Generally, the data were summarized as means, medians (minimum, 25th and 75th percentiles, and maximum) and standard deviations for continuous variables and frequency with percentages for categorical variables. OS and PFS were estimated using the Kaplan-Meier method. Univariate analyses were performed using the log-rank test, and multivariate analyses were performed with the Cox proportional hazards model. SPSS (version 22; IBM Inc., Armonk, NY, USA) was used for all statistical analyses.

\section{Results}

A total of 115 patients were newly diagnosed with glioblastoma between January 2008 and June 2016. Of these, 99 were eligible to be enrolled. Sixteen patients were excluded because they were not treated with CCRT ( $\mathrm{n}=9$ ), did not have histologically proven glioblastoma ( $\mathrm{n}$ $=5)$, or were $<18$ years old $(n=2)$. All eligible patients received CCRT, with a median time from surgical intervention to the start of CCRT of
5.1 weeks (range, 1.4-12.8 weeks). The patients were grouped according to the time to radiotherapy initiation. A total of 21 patients $(21.2 \%)$ started CCRT within 4 weeks after surgery, 32 (32.2\%) 4-6 weeks after surgery, and $46(46.5 \%)>6$ weeks after surgery. The baseline and clinical characteristics of the patients are shown in Table 1.

The median PFS of the patients in these groups was 11.2 months, 7.6 months, and 9.2 months respectively; their median OS was 25 months, 16.9 months, and 20.1 months, respectively (Figures 1 and 2). Multivariate Cox regression analysis, adjusted for age, sex, Eastern Cooperative Oncology Group (ECOG) performance status (PS), the presence of comorbidities, surgical status, and adjuvant chemotherapy showed no difference in OS between the groups (Table 2). In the multivariate analysis, better survival was associated with receiving adjuvant TMZ and a good ECOG PS. Worse survival was associated with an ECOG PS $>2$, the presence of comorbidities, and residual disease (Table 3).

\section{Discussion}

Robust results on the effect of the time to chemoradiotherapy initiation after surgery on clinical outcomes exist for carcinoma of the breast as well as head and neck cancer; the optimal interval between surgery and the first RT session in breast cancer is 8 weeks [14-16]. In head and neck cancers treated with primary surgery, most studies reported a higher probability of locoregional recurrence in patients treated with RT or chemoradiotherapy that was initiated $>6$ weeks after the surgical intervention [17]. However, current data on the effect of the time to CCRT initiation on the outcomes of patients with glioblastoma are limited and conflicting [8]. The effect of the time interval between surgery and the initiation of chemoradiotherapy in glioblastoma has been studied in some retrospective analyses. The lack of an effect of the time interval between surgery and CCRT initiation in our data is in line with some other clinical studies. Several retrospective datasets have shown no effect of this time interval on outcomes in patients with glioblastoma $[10,18,19]$. Moreover, Blumenthal et al. not only found that a treatment delay was not associated with worse survival but also that the group with the longest delay had the best outcome [10]. In contrast, a study by Irwin et al. showed that the risk

\begin{tabular}{|c|c|c|c|c|c|c|c|}
\hline & & \multirow{2}{*}{\multicolumn{2}{|c|}{$\begin{array}{c}<4 \text { weeks } \\
(n=21)\end{array}$}} & \multirow{2}{*}{\multicolumn{2}{|c|}{$\begin{array}{c}\text { 4-6 weeks } \\
(n=32)\end{array}$}} & \multirow{2}{*}{\multicolumn{2}{|c|}{$\begin{array}{c}>6 \text { weeks } \\
(n=46)\end{array}$}} \\
\hline & & & & & & & \\
\hline & & $\mathbf{n}$ & $\%$ & $\mathbf{n}$ & $\%$ & $\mathbf{n}$ & $\%$ \\
\hline \multirow{2}{*}{ Age, years } & Median & 51 & & 47 & & 49 & \\
\hline & $\geq 65$ & 4 & 19 & 2 & 6.3 & 9 & 19.5 \\
\hline \multirow{2}{*}{ Sex } & Male & 13 & 61.9 & 20 & 62.5 & 38 & 82.6 \\
\hline & Female & 8 & 38.1 & 12 & 37.5 & 8 & 17.4 \\
\hline \multirow{2}{*}{ ECOG PS } & $<2$ & 15 & 71.4 & 21 & 65.6 & 36 & 78.2 \\
\hline & $\geq 2$ & 6 & 28.5 & 11 & 34.6 & 10 & 21.7 \\
\hline \multirow{2}{*}{ Comorbidities } & Yes & 7 & 33.3 & 11 & 34.3 & 19 & 41.3 \\
\hline & No & 14 & 66.6 & 21 & 65.6 & 27 & 58.7 \\
\hline \multirow{3}{*}{ Surgical status } & Any resection & 16 & 76.2 & 31 & 96.8 & 41 & 89.1 \\
\hline & Gross resection & 3 & 14.3 & 6 & 18.7 & 5 & 10.9 \\
\hline & Biopsy only & 5 & 23.8 & 1 & 3.1 & 5 & 10.9 \\
\hline \multirow{2}{*}{$\begin{array}{c}\text { Adjuvant } \\
\text { Chemotherapy }\end{array}$} & Yes & 18 & 85.7 & 26 & 81.3 & 33 & 71.7 \\
\hline & No & 3 & 14.2 & 6 & 18.7 & 13 & 28 \\
\hline \multirow{3}{*}{ MGMT status } & Methylated & 0 & 0 & 4 & 12.5 & 5 & 10.8 \\
\hline & Unmethylated & 0 & 0 & 3 & 9.4 & 4 & 8.6 \\
\hline & Unknown & 21 & 100 & 25 & 78.1 & 37 & 80.4 \\
\hline
\end{tabular}

ECOG: Eastern Cooperative Oncology Group; MGMT: O6-methylguanine-DNA-methyltransferase; PS: performance status.

Table 1: Baseline and clinical characteristics of the patients. 


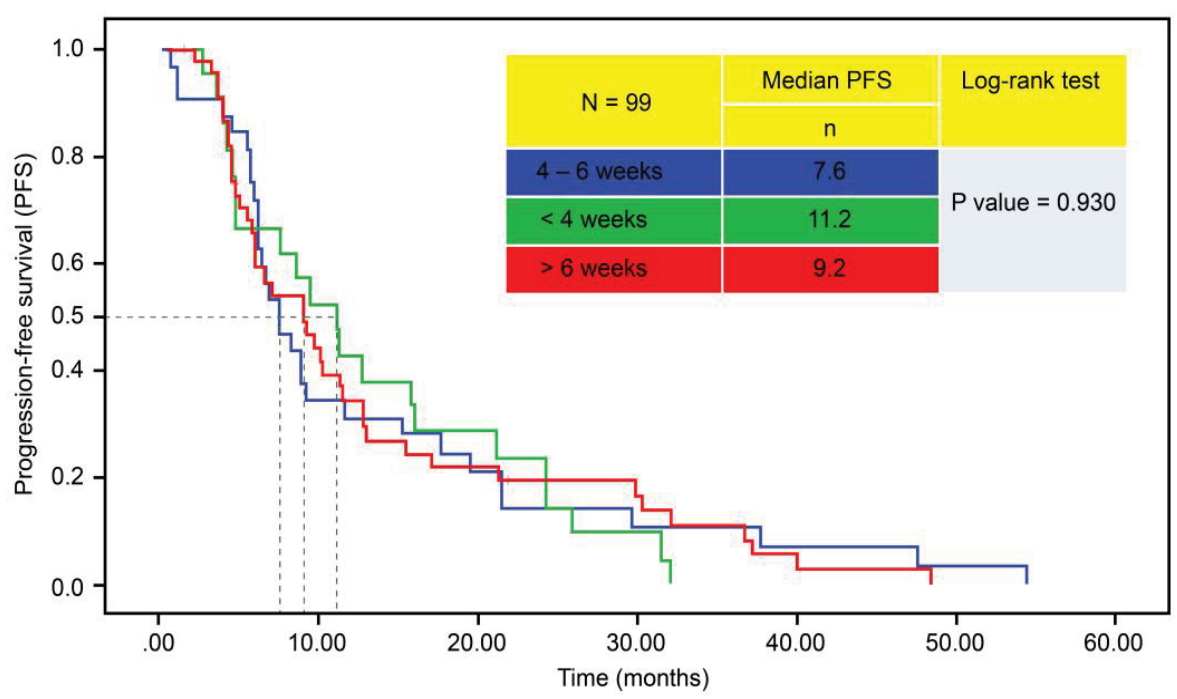

Figure 1: Progression-free survival by time interval to therapy PFS, progression-free survival.

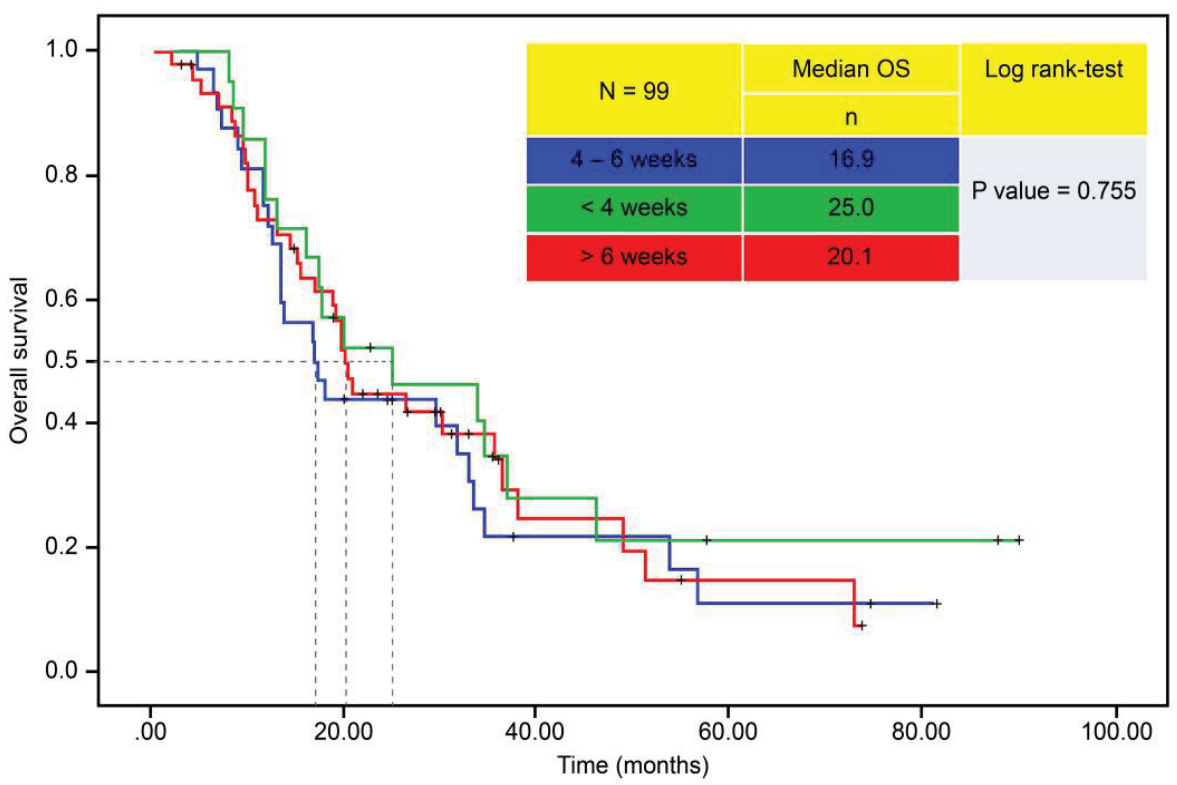

Figure 2: Overall survival by time interval to therapy OS, overall survival.

\begin{tabular}{|c|c|c|c|}
\hline & $(\mathbf{n}=\mathbf{9 9})$ & & \\
\hline Adjusted multivariate analysis $^{*}$ & HR & $95 \% \mathrm{Cl}$ & P value \\
\hline 4-6 weeks & Reference & & \\
\hline <4 weeks & 1.29 & $0.75-2.23$ & 0.36 \\
\hline >6 weeks & 0.76 & $0.39-1.47$ & 0.42 \\
\hline
\end{tabular}

*Multivariate Cox regression adjusted for age; sex, Eastern Cooperative Oncology Group (ECOG) performance status; presence of comorbidities; surgical status; adjuvant chemotherapy. $\mathrm{Cl}$ : confidence interval; HR: hazard ratio.

Table 2: Multivariate analysis of the association between the time to concurrent chemoradiotherapy initiation and overall survival.

of death (as measured by the hazard ratio) increased by $2 \%$ for each day and by $8.9 \%$ for every week the initiation of RT was delayed [13]. To the best of our knowledge, the data presented here are the first to analyze the effect of the time interval between surgery and CCRT on the treatment outcomes of patients with glioblastoma in Saudi Arabia; moreover, this is one of only few studies that examined this question in a clinical and real-life setting. In this study, we showed that the time to the initiation of CCRT after surgery did not significantly affect the patients' outcomes. There are certain limitations to the current study, mainly arising from its retrospective design, the relatively small sample size, the use of data from a single center, and the scarcity of molecular marker data such as MGMT methylation and IDH mutation status. The evidence on the effect of the timing of CCRT on survival outcomes in this study is exclusively retrospective, and there may have been a bias in the clinician's decisions to rush patients who were likely to do poorly with early adjuvant therapy. In conclusion, the present study showed no statistically significant effect of the time to the initiation of CCRT 
Citation: Alhumiqani A, Basulaiman B, Elyamany A, Balbaid A, Altwairgi AK (2018) Impact of the Time to Chemoradiation Initiation in Patients with Glioblastoma. J Integr Oncol 7: 209. doi: 10.4172/2329-6771.1000209

\begin{tabular}{|c|c|c|c|}
\hline ECOG PS & HR & $95 \% \mathrm{Cl}$ & $P$ value \\
\hline$\leq 2$ & 0.51 & $0.30-0.86$ & 0.013 \\
\hline$>2$ & & & \\
\hline \multicolumn{4}{|c|}{ Comorbidities } \\
\hline Yes & 1.76 & $1.08-2.87$ & 0.023 \\
\hline No & & & \\
\hline \multicolumn{4}{|c|}{ Gross resection } \\
\hline No & 2.24 & $1.09-4.59$ & 0.027 \\
\hline Yes & & & \\
\hline \multicolumn{4}{|c|}{ Adjuvant chemotherapy } \\
\hline Yes & 0.5 & $0.28-0.92$ & 0.025 \\
\hline No & & & \\
\hline
\end{tabular}

Table 3: The multivariate analysis of the prognostic factors in patients with glioblastoma.

Cl: confidence interval; ECOG: Eastern Cooperative Oncology Group; HR: hazard ratio; PS: performance status.

on PFS and OS in patients with glioblastoma. Prospective studies are needed to define the optimal timing of CCRT after surgical resection in patients with gliobla-stoma.

\section{Acknowledgements}

We would like to show our gratitude to the King Fahad Medical City (KFMC) for their support of this research. We extend a special thank you to our colleagues in the Departments of Pathology, Radiology, and Nursing for their insight, help, and cooperation.

\section{References}

1. Stupp R, Hegi ME, Mason WP, van den Bent MJ, Taphoorn MJ, et al. (2009) Effects of radiotherapy with concomitant and adjuvant temozolomide versus radiotherapy alone on survival in glioblastoma in a randomised phase III study: 5-year analysis of the EORTC-NCIC trial. Lancet Oncol 10: 459-466.

2. Li J, Wang M, Won M, Shaw EG, Coughlin C, et al. (2011) Validation and simplification of the Radiation Therapy Oncology Group recursive partitioning analysis classification for glioblastoma. Int J Radiat Oncol Biol Phys 81: 623-630.

3. Mirimanoff RO, Gorlia T, Mason W, Van den Bent MJ, Kortmann RD, et al. (2006) Radiotherapy and temozolomide for newly diagnosed glioblastoma: recursive partitioning analysis of the EORTC 26981/22981-NCIC CE3 phase III randomized trial. J Clin Oncol 24: 2563-2569.

4. Shaw EG, Seiferheld W, Scott C, Coughlin C, Leibel S, et al. (2003) Reexamining the radiation therapy oncology group (RTOG) recursive partitioning analysis (RPA) for glioblastoma multiforme (GBM) patients. Int J Radiat Onco Biol Phys 57: S135-S136

5. Bese NS, Sut PA, Ober A (2005) The effect of treatment interruptions in the postoperative irradiation of breast cancer. Oncol 69: 214-223.
6. Thames HD, Kuban D, Levy LB, Horwitz EM, Kupelian P et al. (2010) The role of overall treatment time in the outcome of radiotherapy of prostate cancer: an analysis of biochemical failure in 4839 men treated between 1987 and 1995 Radiother Oncol 96: 6-12.

7. Withers HR, Peters LJ, Taylor JM, Owen JB, Morrison WH, et al. (1995) Loca control of carcinoma of the tonsil by radiation therapy: an analysis of patterns of fractionation in nine institutions. Int J Radiat Oncol Biol Phys 33: 549-562.

8. Lawrence YR, Blumenthal DT, Matceyevsky D, Kanner AA, Bokstein F, et al (2011) Delayed initiation of radiotherapy for glioblastoma: how important is it to push to the front (or the back) of the line? J Neurooncol 105: 17.

9. Seidlitz A, Siepmann T, Lock S, Juratli T, Baumann M, et al. (2015) Impact of waiting time after surgery and overall time of postoperative radiochemotherapy on treatment outcome in glioblastoma multiforme. Radiat Oncol 10: 172.

10. Blumenthal DT, Won M, Mehta MP, Curran WJ, Souhami L, et al. (2009) Short delay in initiation of radiotherapy may not affect outcome of patients with glioblastoma: a secondary analysis from the radiation therapy oncology group database. J Clin Oncol 27: 733-739.

11. Han SJ, Rutledge WC, Molinaro AM, Chang SM, Clarke JL, et al. (2015) The effect of timing of concurrent chemoradiation in patients with newly diagnosed glioblastoma. Neurosurgery $77: 248-253$.

12. Do V, Gebski V, Barton MB (2000) The effect of waiting for radiotherapy for grade III/IV gliomas. Radiother Oncol 57: 131-136.

13. Irwin C, Hunn M, Purdie G, Hamilton D. (2007) Delay in radiotherapy shortens survival in patients with high-grade glioma. J Neurooncol 85: 339-343.

14. Huang J, Barbera L, Brouwers M, Browman G, Mackillop WJ (2003) Does delay in starting treatment affect the outcomes of radiotherapy? A systematic review. J Clin Oncol 21: 555-563.

15. Froud PJ, Mates D, Jackson JS, Phillips N, Andersen S, et al. (2000) Effect of time interval between breast conserving surgery and radiation therapy on ipsilateral breast recurrence. Int J Radiat Oncol Biol Phys 46: 363-372.

16. Ampil FL, Burton GV, Li BD, Mills GM (1999). Radiotherapy with and without chemotherapy after breast conservation surgery for early stage breast cancer: A review of timing. Eur J Gynaecol Oncol 20: 254-257.

17. Bastit L, Blot E, Debourdeau P, Menard J, et al. (2001) Influence of the delay of adjuvant postoperative radiotherapy on relapse and survival in oropharyngeal and hypopharyngeal cancers. Int J Radiat Oncol Biol Phys 49: 139-146.

18. Noel G, Huchet A, Feuvret L, Maire JP, Verrelle P, et al. (2012) Waiting times before initiation of radiotherapy might not affect outcomes for patients with glioblastoma: a French retrospective analysis of patients treated in the era of concomitant temozolomide and radiotherapy. J Neurooncol 109:167-175.

19. Sun MZ, Oh T, Ivan ME, Clark AJ, Safaee M, et al. (2005) Survival impact of time to initiation of chemoradiotherapy after resection of newly diagnosed glioblastoma. J Neurosurg 122: 1144-1150. 